\title{
The presence of NAFLD in nonobese subjects increased the risk of metabolic abnormalities than obese subjects without NAFLD: a population-based cross-sectional study
}

\author{
Ping-Fang Hu ${ }^{1 \#}$, Xin Zeng ${ }^{2 \#}$, Zi-Yuan Zou ${ }^{1,3 \#}$, Wei Tang ${ }^{4 *}$, Yi-Bin Guo ${ }^{5}$, Zong-Li Yuan ${ }^{1}$, Pei-Mei Shi ${ }^{1}$, \\ Yu Tan ${ }^{1}$, Yan Song ${ }^{4}$, Yong-Quan Shi ${ }^{4}$, Wei-Fen Xie ${ }^{1}$
}

${ }^{1}$ Department of Gastroenterology, Changzheng Hospital, Second Military Medical University, Shanghai, China; ${ }^{2}$ Department of Gastroenterology, East Hospital, Tongji University School of Medicine, Shanghai, China; ${ }^{3}$ The First Clinical School, Nanfang Hospital, Southern Medical University, Guangzhou, China; ${ }^{4}$ Department of Endocrinology, Changzheng Hospital, Second Military Medical University, Shanghai, China; ${ }^{5}$ Department of Health Statistics, Second Military Medical University, Shanghai, China

Contributions: (I) Conception and design: PF Hu, X Zeng, ZY Zou, W Tang, YQ Shi, WF Xie; (II) Administrative support: YQ Shi, WF Xie; (III) Provision of study patients: PF Hu, X Zeng, ZY Zou, W Tang, ZL Yuan, PM Shi, Y Tan, Y Song; (IV) Collection and assembly of data: PF Hu, X Zeng, W Tang, Y Song; (V) Data analysis and interpretation: PF Hu, X Zeng, ZY Zou, YB Guo; (VI) Manuscript writing: All authors; (VII) Final approval of manuscript: All authors.

\#These authors contributed equally to this work.

Correspondence to: Wei-Fen Xie. Department of Gastroenterology, Changzheng Hospital, Second Military Medical University, Shanghai 200003, China. Email: weifenxie@medmail.com.cn; Yong-Quan Shi. Department of Endocrinology, Changzheng Hospital, Second Military Medical University, Shanghai 200003, China. Email: young.stone@163.com.

Background: With lifestyle modification and over-nutrition, the prevalence of nonalcoholic fatty liver disease (NAFLD) has been increasing annually. Here we aimed to assess the updated prevalence of NAFLD, and to evaluate the association of NAFLD with metabolic abnormalities according to gender, body mass index and age.

Methods: A population-based cross-sectional study was conducted in Shanghai from December 2016 to July 2017. With a three-stage stratified sampling strategy, 3,717 eligible participants were enrolled for the analysis.

Results: In total, 1,217 subjects (32.7\%) had NAFLD. Among them, 400 (16.3\%) of the nonobese and $817(65.0 \%)$ of the obese subjects had NAFLD. The prevalence of NAFLD was increased according to the quartiles of age and waist circumference (WC) in the nonobese subjects. Females with nonobese NAFLD had 1.6-, 2.6-, 2.0-, 2.3- and 3.3-fold higher risks for metabolic syndrome, diabetes mellitus, hyperglycemia, hypertriglycerdemia (high TG) and low high-density lipoprotein cholesterol than obese subjects without NAFLD, respectively. Males had comparable metabolic profiles in both groups, except for a 2.0-fold higher risk of high TG in nonobese NAFLD subjects compared with obese subjects without NAFLD. More impressively, the homeostasis metabolic assessment insulin resistance index was comparable between the two groups.

Conclusions: The increase of age and WC had significant impact on the risk of NAFLD in nonobese subjects. The presence of NAFLD in nonobese subjects increased the risk of metabolic diseases than obese subjects without NAFLD, especially in female.

Keywords: Non-alcoholic fatty liver disease (NAFLD); obesity; metabolic syndrome (MetS); insulin resistance

Submitted Jan 28, 2020. Accepted for publication Mar 18, 2020.

doi: $10.21037 / \mathrm{hbsn}-20-263$

View this article at: https://dx.doi.org/10.21037/hbsn-20-263 


\section{Introduction}

Non-alcoholic fatty liver disease (NAFLD) is the most common chronic liver disease worldwide, affecting approximately $25 \%$ of the adult population globally (1). NAFLD was historically considered as a disease of the industrialized world, and the epidemiology has been well characterized in western countries $(2,3)$. With the rapid urbanization and sedentary lifestyle, the prevalence of NAFLD has been growing during the past decades in many part of Asia $(3,4)$. It has been reported that the prevalence of NAFLD in China has increased from $15 \%$ to $25 \%$ over the past ten years $(5,6)$, whereas most of the data are based on hospital surveys. There is a paucity of updated epidemiological data based on the communities' survey in China.

Unhealthy lifestyles play an essential role in the development and progression of NAFLD. Currently, there is no effective drug therapy for NAFLD (7). And lifestyle interventions including dietary modification and physical activity remain the first-line treatment for NAFLD to date $(7,8)$. Recently, Mediterranean diet has even been suggested for NAFLD patients as its harmonic combination of several foods with antioxidant and anti-inflammatory properties (9).

NAFLD used to be connected with obesity, whereas increasing evidence has indicated that NAFLD can occur in subjects with normal weight (lean) or those who are overweight as well, which is known as lean NAFLD or nonobese NAFLD (10). The reported prevalence of lean/ nonobese NAFLD in Asia varied differently, ranging from $4.1 \%$ to $27.4 \%$ (11-14). And it can also occur in lean Caucasian subjects (15). Histology evaluation revealed that nonobese NAFLD had less severe liver damage at presentation than that of the subjects with obese NAFLD $(11,16)$. Leung et al. (17) also reported that patients with nonobese NAFLD had a better prognosis than those with obese NAFLD after a median of 49 months followup. Nevertheless, up to $10.5-25.0 \%$ of individuals with nonobese NAFLD had advanced fibrosis, which was related with an increased risk of liver-related mortality (17-19). Thus, nonobese NAFLD might not be such a benign condition as previously thought (20).

NAFLD was connected with metabolic diseases (21) and even considered as a metabolic manifestation in the liver (22). It is now widely accepted that, like obese NAFLD, subjects with lean NAFLD also had altered glucolipid metabolism and metabolic profile $(23,24)$. A retrospective cohort study showed that approximately one-fifth of the lean NAFLD patients had carotid atherosclerosis (25). In addition, nonobese NAFLD was associated with a higher risk of metabolic diseases compared to obese NAFLD (26). However, previous studies have comprehensively compared the characteristics of lean/nonobese NAFLD with those of lean healthy or overweight/obese NAFLD $(16,23)$, whereas there are no data on the differences between lean/nonobese subjects with NAFLD and obese subjects without NAFLD.

Hence, in this study, we provided updated prevalence data for NAFLD in subjects in Shanghai according to a large population-based investigation. We found that nonobese subjects were more vulnerable to NAFLD with the increase of age and waist circumference (WC) than the obese subjects. In addition, nonobese subjects with NAFLD had a higher risk of metabolic diseases than obese subjects without NAFLD, especially in female. We present the following article in accordance with the STROBE reporting checklist (available at https://hbsn.amegroups.com/article/view/10.21037/hbsn$20-263 / \mathrm{rc})$.

\section{Methods}

\section{Ethical statement}

The study was conducted in accordance with the Declaration of Helsinki (as revised in 2013). Written informed consent was obtained from all participants in the study. The study protocol was approved by the Shanghai Changzheng Hospital Institutional Ethics Committee (2016SL039).

\section{Study design and participants}

It was a population-based, cross-sectional health survey of the inhabitants in Shanghai, which was composed of 18 districts and 242 community health service centers. A three-stage stratified sampling strategy was used to obtain a representative sample of the population in Shanghai from December 2016 to July 2017. First, 10 representative community health service centers were selected randomly based on the living area, of which 4 were from the urban areas, 3 were from the urban fringe areas, and 3 were from the suburbs. Second, 10 neighborhood communities were randomly selected from each community health service center. Third, the household demographics were obtained from the neighborhood communities, and one subject was selected from each household randomly. Inclusion criteria consisted of subjects without serious chronic diseases who lived in the selected community for more than six months and were aged between 18 and 65 years. Exclusion criteria 


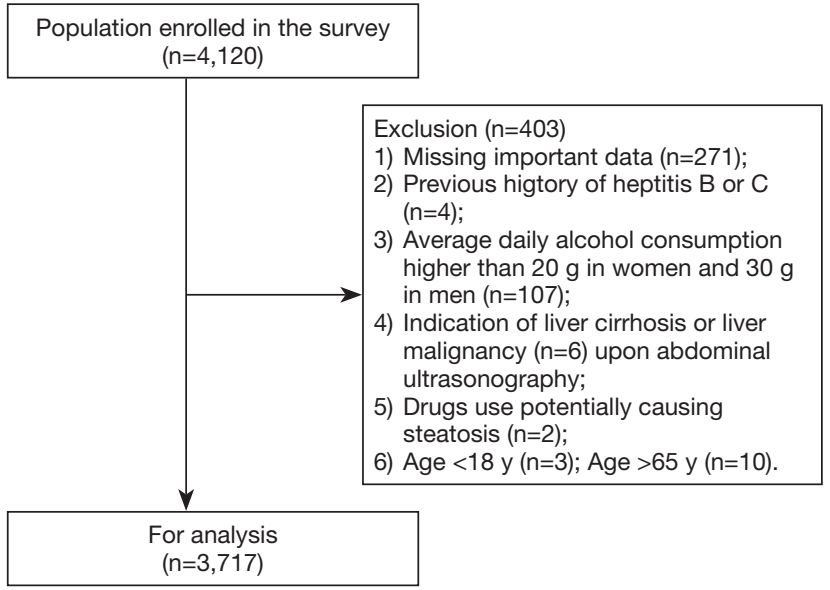

Figure 1 Flowchart of the enrolled subjects.

included the following: pregnancy, fracture, severe mental disorder or dementia, action inconvenience, hepatitis B and C, tuberculosis, human immunodeficiency virus or other infectious disease. Finally, 4,193 subjects were selected and invited to participate in the study, and 4,120 subjects completed the study. The overall response rate was $98.3 \%$.

For this study, we excluded individuals as follows: (I) missing height, weight, WC or abdominal ultrasonographic results ( $\mathrm{n}=42$ ); (II) missing blood test results ( $\mathrm{n}=229$ ); (III) previous history of hepatitis B or C infection, Wilson's disease or autoimmune hepatitis on the questionnaire $(n=4)$; (IV) average daily alcohol consumption higher than $20 \mathrm{~g}$ in women and $30 \mathrm{~g}$ in men $(\mathrm{n}=107) ;(\mathrm{V})$ indication of liver cirrhosis $(\mathrm{n}=5)$ or liver malignancy $(\mathrm{n}=1)$ upon abdominal ultrasonography; (VI) subjects using drugs potentially causing steatosis ( $\mathrm{n}=2$, prednisolone); and (VII) age $<18$ years $(n=3)$ and age $>65$ years $(n=10)$. Ultimately, a total of 3,717 subjects were included in our data analysis (Figure 1).

\section{Data acquisition}

A complete questionnaire survey, anthropometric measurements, abdominal ultrasound and blood samples were collected at each community health service center. Individuals were interviewed privately by well-trained medical professionals to complete a questionnaire including date of birth, sex, education level, occupation, medical history of diseases, menstrual history for females, alcohol consumption (type, amount and frequency), smoking status and medication use. Individuals were categorized into current, former, or never-smokers. Drinking status was divided into never, occasional drinker $(<10 \mathrm{~g} / \mathrm{d})$ and frequent drinker $(\geq 10 \mathrm{~g} / \mathrm{d})$.

\section{Anthropometric measurement}

Height, weight, WC and blood pressure (BP) were measured by trained personnel with a standardized protocol. Body mass index (BMI) was calculated by dividing weight $(\mathrm{kg})$ by the square of height $\left(\mathrm{kg} / \mathrm{m}^{2}\right)$. WC was measured horizontally at the level of the umbilicus. Prior to measuring BP, the subjects were asked to rest for $5 \mathrm{~min}$.

\section{Abdominal ultrasonography}

Fatty liver was determined by the ultrasonographic measurements with a $3.5 \mathrm{MHz}$ probe by experienced sonographers who were blinded to the clinical findings of the subjects. The diagnosis of fatty liver depended on a diffuse increase in the fine echoes in the liver parenchyma compared with that of the spleen or kidney, liver brightness with deep attenuation and vessel blurring.

\section{Laboratory analyses}

Blood samples were obtained in the morning with an empty stomach after an overnight fast of at least $12 \mathrm{~h}$. Laboratory evaluations included liver function test [total bilirubin (TB), direct bilirubin (DB), alanine aminotransferase (ALT), aspartate aminotransferase (AST), $\gamma$-glutamyl transpeptidase (GGT), albumin, total protein (TP), and alkaline phosphatase (AKP)], fasting blood glucose (FBG), fasting insulin (Ins), triglycerides (TG), total cholesterol (TC), high-density lipoprotein cholesterol (HDL), low-density lipoprotein cholesterol (LDL), hemoglobin A1c (HbA1C), uric acid (SUA) and creatinine (SCr). Except subjects with a validated history of diabetes mellitus (DM), all individuals underwent a $75-\mathrm{g}$ oral glucose tolerance test. The blood glucose level was evaluated $2 \mathrm{~h}$ later ( $2 \mathrm{hFBG}$ ).

\section{Definitions}

The subjects were categorized into the nonobese (BMI $<25)$ and obese $(\mathrm{BMI} \geq 25)$. Accordingly, all subjects were divided into four groups: nonobese non-NAFLD (subjects without NAFLD), nonobese NAFLD, obese non-NAFLD and obese NAFLD. Metabolic syndrome (MetS) was defined according to the Chinese Diabetes Society criteria (27), which required the presence of three 
or more of the following components: (I) abdominal obesity: $W C \geq 90 \mathrm{~cm}$ for men and $W C \geq 85 \mathrm{~cm}$ for women; (II) high BP (HBP): systolic blood pressure (SBP) $\geq 130 \mathrm{mmHg}$, and/or diastolic blood pressure (DBP) $\geq 85 \mathrm{mmHg}$, or previously diagnosed as hypertension and under treatment; (III) hypertriglyceridemia (high TG): fasting TG $\geq 1.7 \mathrm{mmol} / \mathrm{L}$, or under specific treatment for this lipid abnormality; (IV) low HDL: fasting HDL $<1.04 \mathrm{mmol} / \mathrm{L}$; and (V) hyperglycemia: $\mathrm{FBG} \geq 6.1 \mathrm{mmol} / \mathrm{L}$, or $2 \mathrm{hFBG} \geq 7.8 \mathrm{mmol} / \mathrm{L}$ during oral glucose tolerance test, or previously diagnosed as DM and treatment with antidiabetic drugs.

$\mathrm{DM}$ was defined as $\mathrm{FBG} \geq 7.0 \mathrm{mmol} / \mathrm{L}$, or $2 \mathrm{hFBG}$ $\geq 11.1 \mathrm{mmol} / \mathrm{L}$ during an oral glucose tolerance test, or $\mathrm{HbA1C} \geq 6.5 \%$. The homeostasis metabolic assessment insulin resistance index (HOMA-IR) was used to define insulin resistance and was calculated with the following formula: HOMA-IR = FBG $(\mathrm{mmol} / \mathrm{L}) \times \mathrm{Ins}(\mathrm{mU} / \mathrm{L}) / 22.5$.

\section{Statistical analysis}

Statistical analysis was performed using the SPSS software package version 18.0 for Windows (SPSS, Chicago, IL). Continuous variables are described as the mean \pm SD and compared with Student's $t$-test or the Mann-Whitney $\mathrm{U}$ test, depending on the normality of the data. Categorical variables are reported as the subject number with percentage (\%) and compared using the $\chi^{2}$ test between groups. We presented these data as odds ratios (ORs) of the risk of NAFLD according to the quartiles of age and WC in both genders using a logistic regression model, adjusting for potentially confounding variables, including education, living area, smoking, drinking and menopause status for females. Meanwhile, the associations of the presence of NAFLD in nonobese and obese subjects with metabolic diseases including MetS, DM, high TG and low HDL are presented as ORs [95\% confidence interval (CI)], using a logistic regression model, adjusting for education, living area, smoking, drinking and menopause status for female. A twotailed $\mathrm{P}$ value $<0.05$ was considered to be statistically significant.

\section{Results}

\section{Basic characteristics of the enrolled participants}

The clinical characteristics of the participants are shown in Table 1. In total, 3,717 subjects (1,577 males and 2,140 females, with a mean age of $41.9 \pm 12.1$ years) were enrolled in the study. Among them, 1,217 (32.7\%) had NAFLD, $1,178(31.7 \%)$ had abdominal obesity, 1,257 (33.8\%) had obesity, 751 (20.2\%) had MetS, 311 (8.4\%) had DM, and $929(25.0 \%)$ had high TG. NAFLD was male predominant, with $726(46.0 \%)$ males and 491 (22.9\%) females having NAFLD. And subjects with NAFLD were older than those without NAFLD $(45.1 \pm 11.4$ vs. $40.4 \pm 12.1$ years, $\mathrm{P}<0.001)$. Compared with subjects without NAFLD, the mean levels of BMI, WC, ALT, AST, GGT, AKP, SUA, SCr, FBG, 2hFBG, Ins, HOMA-IR, HbA1C, TC, TG and LDL in subjects with NAFLD were significantly higher, whereas the level of HDL was significantly lower both in males and females. Subjects with NAFLD had significantly higher percentages of abdominal obesity, MetS, DM, high TG, low HDL and HBP than those of the subjects without NAFLD in both genders. There was no difference in the living area distribution between subjects with and without NAFLD. Interestingly, the risk of NAFLD decreased in female subjects with higher levels of education. Compared with females with illiteracy/primary school level of education, females with junior/senior high school and a bachelor/ higher level of education had ORs of 0.69 (0.45-1.04, $\mathrm{P}=0.074)$ and $0.28(0.18-0.44, \mathrm{P}<0.001)$, respectively. However, this difference was not seen in males.

\section{Characteristics of the participants with and without NAFLD stratified by gender and BMI}

To investigate the characteristics of the nonobese and obese NAFLD separately, we divided the study population into the nonobese group (BMI <25) and obese group (BMI $\geq 25$ ). As shown in Table S1, 400 (16.3\%) of the nonobese subjects had NAFLD (nonobese NAFLD), whereas 817 (65.0\%) of the obese subjects had NAFLD (obese NAFLD, $\mathrm{P}<0.001$ ). In both males and females, the laboratory values for SUA, FBG, 2hFBG, HbA1C, HOMA-IR and the prevalence of abdominal obesity, MetS, DM, high TG, low HDL and HBP were significantly higher in the NAFLD group than those in the controls. In addition, the presence of NAFLD significantly increased the risk of metabolic diseases including MetS, DM, high TG and low HDL in both genders, irrespective of the BMI categories (Figure S1).

\section{Nonobese subjects with NAFLD bave more severe metabolic abnormalities than obese subjects without NAFLD}

It is now well known that obesity is an essential component of MetS (28). To explore the influence of obesity and 
Table 1 Characteristics of the enrolled participants



Table 1 (Continued) 
Table 1 (Continued)

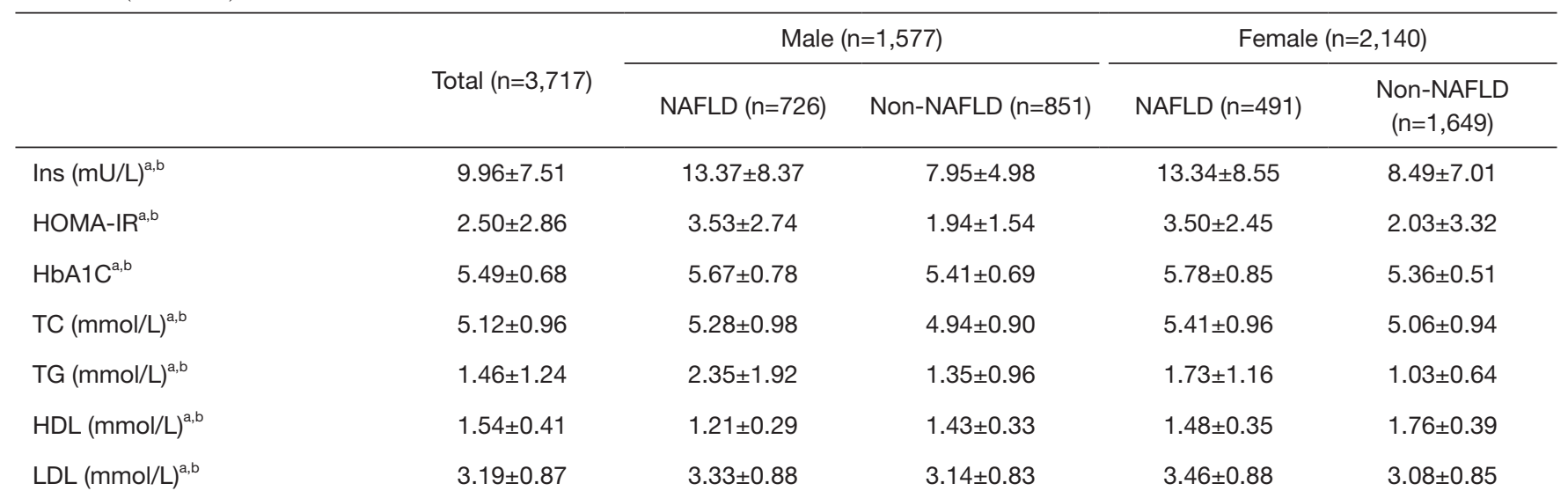

Data are presented as mean $\pm \mathrm{SD}$ or $\mathrm{n}(\%)$. ${ }^{\mathrm{a}}$, component with a $\mathrm{P}$ value of $<0.05$ in the male group; ${ }^{\mathrm{b}}$, component with a $\mathrm{P}$ value of $<0.05$ in the female group. NAFLD, nonalcoholic fatty liver disease; SD, standard deviation; BMI, body mass index; WC, waist circumference; MetS, metabolic syndrome; DM, diabetes mellitus; high TG, hypertriglyceridemia; HDL, high-density lipoprotein cholesterol; HBP, high blood pressure; ALT, alanine aminotransferase; AST, asparate aminotransferase; GGT, $\gamma$-glutamyl transpeptidase; TB, total bilirubin; Alb, albumin; AKP, alkaline phosphatase; TP, total protein; SUA, serum uric acid; SCr, serum creatinine; FBG, fasting blood glucose; 2hFBG, 2-hour postprandial blood glucose; Ins, insulin; HOMA-IR, homeostasis metabolic assessment insulin resistance index; HbA1C, hemoglobin A1c; TC, total cholesterol; TG, triglycerides; LDL, low-density lipoprotein cholesterol.

NAFLD on the risk of metabolic diseases, we exclusively compared the metabolic profiles between the nonobese subjects with NAFLD (nonobese NAFLD) and obese subjects without NAFLD (obese non-NAFLD; Table 2 and Figure 2). Subjects in the obese non-NAFLD group had much higher percentages of abdominal obesity than those in the nonobese NAFLD group in both genders. The HOMAIR indexes were comparable between the two groups in both genders. To our astonishment, females with nonobese NAFLD had significantly higher prevalence of MetS, DM, hyperglycemia, high TG, low HDL and higher levels of SUA, FBG, 2hFBG, HbA1C than obese female without NAFLD. After adjusting for potentially confounding variables including education, living area, smoking, drinking and menopause status, females with nonobese NAFLD had 1.6-, 2.6-, 2.0-, 2.3- and 3.3-fold higher risks for MetS, DM, hyperglycemia, high TG and low HDL than the obese non-NAFLD group, respectively. In males, there were comparable metabolic profiles in both groups, except for a 2.0-fold higher risk of high TG in the nonobese NAFLD group compared with the obese non-NAFLD subjects.

\section{Nonobese subjects are more vulnerable to NAFLD with the increase of age and WC than the obese subjects}

As shown in Table S1, the mean ages of nonobese subjects with NAFLD were markedly older than those of nonobese subjects without NAFLD in both genders $(44.5 \pm 11.8 \mathrm{vs}$. $39.9 \pm 12.8$ years in males; $48.9 \pm 10.8$ vs. $39.5 \pm 11.5$ years in females, $\mathrm{P}<0.001)$. In addition, we evaluated the prevalence of NAFLD according to age quartiles (Figure 3). Overall, the prevalence of NAFLD was increased with age in the nonobese subjects in both genders. The prevalence of NAFLD was much higher in nonobese males compared to nonobese females in the first three age quartiles, whereas it was almost similar in the fourth age quartile. Meanwhile, males and females showed a disparate prevalence profile with age. In the nonobese males, there was an abrupt increase in prevalence in the second quartile of age (3241 years), whereas the prevalence was at a comparable level thereafter. Compared with the first age quartile (1831 years), nonobese males had a 2.3-, 2.7- and 3.0-fold increased risk of NAFLD for the second (32-41 years), the third (42-53 years) and the fourth (54-65 years) age quartiles, respectively (Table 3). Conversely, in the nonobese females, there was a steady growth among the first three age quartiles. However, the prevalence was dramatically increased when older than 54. Compared with the first age quartile, nonobese females had a 2.8-, 3.5- and 11.2fold increased risk of NAFLD with age, respectively. After adjusting for education level, living area, smoking, drinking status and menopause status, this trend became slightly 
Table 2 Comparison of the metabolic profiles between nonobese subjects with NAFLD (nonobese NAFLD) and obese subjects without NAFLD (obese non-NAFLD) for both genders



Data are presented as $\mathrm{n}(\%)$ or mean \pm SD. NAFLD, nonalcoholic fatty liver disease; SD, standard deviation; BMI, body mass index; MetS, metabolic syndrome; DM, diabetes mellitus; high TG, hypertriglyceridemia; HDL, high-density lipoprotein cholesterol; HBP, high blood pressure; SUA, serum uric acid; FPG, fasting blood glucose; 2hFPG, 2-hour postprandial blood glucose; HOMA-IR, homeostasis metabolic assessment insulin resistance index; HbA1C, hemoglobin A1c. 




Figure 2 Nonobese with NAFLD had higher risks of metabolic diseases than obese subjects without NAFLD. The subjects were classified into obese (BMI $\geq 25$ ) or nonobese (BMI <25) according to BMI. The risk for metabolic diseases including metabolic syndrome (MetS), diabetes mellitus (DM), hyperglycemia, hypertriglyceridemia (high TG), low high-density lipoprotein cholesterol (low HDL) and high blood pressure (HBP) were compared between nonobese NAFLD and obese non-NAFLD subjects using a logistic regression model, adjusting for potentially confounding variables, including education, living area, smoking, drinking status and menopause status for females. Data are presented as the odds ratio (OR, 95\% CI).

attenuated (ORs of 2.7, 3.0 and 8.1, respectively, $\mathrm{P} \leq 0.001$ ). Nevertheless, the differences in the risk of NAFLD among age quartiles were not remarkable in obese females.

In addition, we elucidated the risk of NAFLD according to WC quartiles as well (Table 3). In both genders, it revealed an abrupt increased risk of NAFLD with WC expansion in the nonobese subjects. Compared with the first WC quartile, there was a 2.3-, 4.1- and 7.4-fold higher risk of NAFLD in the nonobese males, and a 4.9-, 12.0and 19.4-fold higher risk of NAFLD in nonobese females with the increase of WC, respectively, after adjusting for education, living area, smoking, drinking status and menopause status for females. In contrast, the increase of WC had little influence on the risk of NAFLD in the obese females. And there was a much lower risk increment as the expansion of WC in the obese males as well.

\section{The existence of NAFLD elevated the risk for metabolic abnormalities in the youtb}

As shown in Figure S1, the risks for metabolic diseases were increased in the nonobese subjects than the obese with NAFLD in both genders. Moreover, we investigated the influence of age on the relation between NAFLD and metabolic diseases as well. And we found that the presence of NAFLD in the youth ( $<45$ years) had significantly higher risks for MetS, DM, hyperglycemia, high TG, low HDL and HBP than the middle-aged and elderly subjects with NAFLD ( $\geq 45$ years, Figure S2). Those results implied that an incidental finding of NAFLD in the nonobese or 



Figure 3 Prevalence of nonalcoholic fatty liver disease (NAFLD) in nonobese (BMI <25) and obese (BMI $\geq 25)$ subjects according to age quartiles.

Table 3 The risk for prevalence of NAFLD in nonobese and obese subjects among different age and WC quartiles for both genders

\begin{tabular}{|c|c|c|c|c|}
\hline & \multicolumn{2}{|c|}{ Nonobese (BMI <25) } & \multicolumn{2}{|c|}{ Obese (BMI $\geq 25)$} \\
\hline \multicolumn{5}{|l|}{ Male } \\
\hline \multicolumn{5}{|l|}{ Age quartile } \\
\hline $18-31$ & Reference & Reference & Reference & Reference \\
\hline $42-53$ & $2.7(1.7-4.3)^{\mathrm{a}}$ & $2.7(1.6-4.4)^{\mathrm{a}}$ & $2.1(1.3-3.4)^{b}$ & $2.0(1.2-3.3)^{\mathrm{C}}$ \\
\hline $54-65$ & $3.0(1.9-4.8)^{\mathrm{a}}$ & $3.1(1.9-5.3)^{\mathrm{a}}$ & $1.0(0.7-1.6)$ & $1.0(0.6-1.6)$ \\
\hline \multicolumn{5}{|l|}{ WC quartile } \\
\hline$\leq 82.0$ & Reference & Reference & Reference & Reference \\
\hline \multicolumn{5}{|l|}{ Female } \\
\hline \multicolumn{5}{|l|}{ Age quartile } \\
\hline $18-31$ & Reference & Reference & Reference & Reference \\
\hline $32-41$ & $2.8(1.5-5.1)^{\mathrm{a}}$ & $2.7(1.5-4.9)^{\mathrm{a}}$ & $1.2(0.7-2.2)$ & $1.3(0.7-2.2)$ \\
\hline $42-53$ & $3.5(1.9-6.3)^{\mathrm{a}}$ & $3.0(1.6-6.0)^{a}$ & $1.4(0.8-2.4)$ & $1.5(0.8-2.7)$ \\
\hline $54-65$ & $11.2(6.4-19.4)^{a}$ & $8.1(3.6-18.0)^{a}$ & $1.7(1.0-2.9)^{\mathrm{c}}$ & $1.9(0.9-4.1)$ \\
\hline \multicolumn{5}{|l|}{ WC quartile } \\
\hline
\end{tabular}

Data are presented as the odds ratio $(95 \% \mathrm{Cl}) .{ }^{\mathrm{a}} \mathrm{P} \leq 0.001 ;{ }^{\mathrm{b}} \mathrm{P} \leq 0.01 ;{ }^{\mathrm{C}} \mathrm{P} \leq 0.05$. Model 1 , unadjusted; Model 2, adjusted for education, living area, smoking, drinking status and menopause status for females. NAFLD, nonalcoholic fatty liver disease; BMI, body mass index; WC, waist circumference; $\mathrm{Cl}$, confidence interval. 
Table 4 The risk for prevalence of NAFLD in nonobese and obese subjects with metabolic diseases

\begin{tabular}{|c|c|c|c|c|}
\hline & \multicolumn{2}{|c|}{ Nonobese (BMI <25) } & \multicolumn{2}{|c|}{ Obese $(\mathrm{BMI} \geq 25)$} \\
\hline \multicolumn{5}{|l|}{ Male } \\
\hline MetS & $5.0(3.2-7.4)^{\mathrm{a}}$ & $4.9(3.2-7.4)^{\mathrm{a}}$ & $3.5(2.4-4.9)^{\mathrm{a}}$ & $3.6(2.5-5.1)^{a}$ \\
\hline Abdominal obesity & $3.5(2.3-5.1)^{\mathrm{a}}$ & $3.4(2.3-5.1)^{\mathrm{a}}$ & $3.2(2.3-4.6)^{\mathrm{a}}$ & $3.2(2.3-4.6)^{\mathrm{a}}$ \\
\hline High TG & $4.7(3.3-6.7)^{\mathrm{a}}$ & $4.6(3.3-6.5)^{\mathrm{a}}$ & $2.9(2.0-4.0)^{a}$ & $3.0(2.1-4.2)^{a}$ \\
\hline Low HDL & $2.7(1.7-4.4)^{\mathrm{a}}$ & $2.9(1.8-4.6)^{\mathrm{a}}$ & $2.2(1.4-3.3)^{\mathrm{a}}$ & $2.2(1.4-3.3)^{a}$ \\
\hline \multicolumn{5}{|l|}{ Female } \\
\hline MetS & $7.9(5.1-12.3)^{a}$ & $5.0(3.2-8.0)^{\mathrm{a}}$ & $5.2(3.4-8.0)^{\mathrm{a}}$ & $5.5(3.5-8.6)^{\mathrm{a}}$ \\
\hline High TG & $5.6(4.0-8.0)^{\mathrm{a}}$ & $4.5(3.1-6.4)^{\mathrm{a}}$ & $2.9(1.9-4.4)^{a}$ & $2.8(1.9-4.3)^{a}$ \\
\hline Low HDL & $5.6(3.0-10.3)^{\mathrm{a}}$ & $5.0(2.6-9.5)^{\mathrm{a}}$ & $3.3(1.4-7.7)^{\mathrm{b}}$ & $3.3(1.4-7.8)^{\mathrm{b}}$ \\
\hline
\end{tabular}

Data are presented as the odds ratio $(95 \% \mathrm{Cl}) .{ }^{\mathrm{a}} \mathrm{P} \leq 0.001 ;{ }^{\mathrm{b}} \mathrm{P} \leq 0.01$. Model 1 , unadjusted; Model 2, adjusted for education, living area, smoking, drinking status and menopause status for females. NAFLD, nonalcoholic fatty liver disease; BMI, body mass index; MetS, metabolic syndrome; DM, diabetes mellitus; high TG, hypertriglyceridemia; HDL, high-density lipoprotein cholesterol.

the youth should be further monitored to rule out the possibilities of metabolic diseases.

\section{Metabolic disorders have a higher impact on the risk of NAFLD in the nonobese subjects compared to the obese}

Subsequently, we evaluated the impact of each metabolic component on the risk of NAFLD according to gender and BMI (Table 4). The presence of MetS, abdominal obesity, DM, high TG and low HDL remarkably increased the risk of NAFLD in obese subjects, with ORs of 3.6, 3.2, 2.3, 3.0 and 2.2 in males and 5.5, 2.3, 2.7, 2.8 and 3.3 in females, respectively. The risks were also considerable in the nonobese subjects, with ORs of 4.9, 3.4, 3.2, 4.6 and 2.9 in males and 5.0, 3.9, 5.0, 4.5 and 5.0 in females, respectively. There was a trend wherein the association of metabolic disorders with NAFLD was more considerable in the nonobese subjects, although it was not statistically significant.

\section{Discussion}

The latest epidemiological data regarding NAFLD in Shanghai were reported in 2005, and it was approximately
$15.35 \%(29)$. Within that study, the authors selected participants from only 2 districts (Yangpu and Pudong New District) of Shanghai, which were an urban and urban fringe area, respectively. To estimate the updated prevalence of NAFLD in the general population of Shanghai, we randomly selected 10 representative districts with a threestep sampling strategy in our study. Most importantly, we conducted our epidemiological surveys based on each community health service center and all of the data were acquired by well-trained professionals with a standardized protocol. According to our study, the prevalence of NAFLD in Shanghai was as high as $32.7 \%$. Meanwhile, the prevalence of NAFLD was comparable to that of obesity (33.8\%) and abdominal obesity (31.7\%), but much higher than that of MetS (20.2\%) and DM (8.4\%).

The status of economy and the living standard have dramatically improved over the past decades in China. As a modern city, western diet diffusion, over-nutrition, sedentary lifestyle and lack of physical activity all substantially contribute to the accelerated prevalence of NAFLD and the related diseases in Shanghai. Nowadays, it has become a serious public health issue which suggests an urgent need to develop and implement a national preventive program for the early detection and treatment. 
Accumulating evidence has indicated that obesity is strongly associated with NAFLD (1). However, mice impairing fat-storage ability in adipocytes showed severe nonobese NAFLD under high fat diet circumstances (30). And an increasing number of clinical studies have also indicated that nonobese subjects could have NAFLD (lean/ nonobese NAFLD) (23). Xu et al. (12) reported that the prevalence of nonobese NAFLD was $7.27 \%$ in Zhejiang, China. Nevertheless, the subjects in that study were employees from the same company. Therefore, that data could not be generalized to the whole population. Here, we revealed that the prevalence of nonobese NAFLD was up to $16.3 \%$ in the nonobese population of Shanghai. This data strongly suggested that nonobese subjects should be screened for NAFLD as well.

The available effective treatment for NAFLD was weight loss through lifestyle modification (8). Lean NAFLD subjects, who had normal weight, also showed improvement of NAFLD from weight reduction (31), and the amount of weight loss needed to achieve remission was less than that needed for obese patients (32). However, doctors should not rely on weight loss alone to target people who would benefit from lifestyle modification. Shao et al. (33) reported that WC was strongly predictive of disease severity among lean, overweight and obese patients with NAFLD. And normal weight subjects with upper-normal WC had an elevated risk of MetS (34). Recently, Sun et al. (35) even showed that normal weight central obesity was associated with higher risk of all-cause and cause-specific mortality compared with normal weight without central obesity among postmenopausal women. In our study, we found that nonobese subjects were more vulnerable to NAFLD as the increase of WC compared to the obese. In this aspect, it might be attributed to the distribution of adipose tissue, as central adiposity has been reported to be associated with greater systemic inflammation (36). Therefore, maintaining a stable WC seemed to be extremely important for the prevention of NAFLD in nonobese subjects.

It is well known that the prevalence of NAFLD appeared to increase with age $(12,37)$. Notably, this trend was only revealed in nonobese subjects from our study. More importantly, after being stratified by gender, we found that nonobese females had the highest risk of NAFLD with age, and the prevalence of NAFLD was dramatically increased at the fourth age quartile (54-65 years). The prevalence of NAFLD in males and females was even similar when older than 54 years. These results might be ascribed to the protective effects of estrogen against NAFLD (38). On the contrary, the obese females did not have an increased risk of NAFLD at all as getting older, which might be due to the compensatory effect of peripheral estrogen produced by excessive fat (39). In this respect, estrogen was also shown to protect against NAFLD-related fibrogenesis (40). Hormone replacement therapy (HRT) was demonstrated to decrease liver enzyme levels in postmenopausal women with DM and presumed NAFLD compared to placebo controls (41). Whether HRT was effective in the treatment of NAFLD or not and the targeted patients need further clinical studies. According to our study, it at least hinted that female, in particular the nonobese, should be monitored for suspected NAFLD as they get older. On another line, a higher education level was associated with a lower risk of NAFLD in females from our study. In view of the current high prevalence of NAFLD and metabolic diseases, we propose that health education regarding lifestyle modification and the harm of NAFLD might be necessary and useful to lower the prevalence of NAFLD.

NAFLD is considered as a metabolic manifestation in the liver and has even been identified as a precursor of MetS $(22,42)$. The presence of NAFLD significantly increased the risk of metabolic diseases. Vice versa, studies have also indicated that metabolic diseases including abdominal obesity, DM, high TG, low HDL and HBP, exacerbated the progression of NAFLD and related kidney dysfunction, irrespective of being obese or not (19). Furthermore, Fukuda et al. (43) found that, compared with the normal weight non-NAFLD group, lean NAFLD had a 3.59-fold increased risk of incident DM after more than 10 years of follow-up, whereas the hazard ratio of overweight subjects without NAFLD was only 1.99. Herein, we found that the existence of NAFLD had an increased risk of MetS, DM, High TG and low HDL, independently from obesity. More importantly, we found that nonobese subjects with NAFLD even had a higher risk of MetS, DM, high TG and low HDL than obese subjects without NAFLD, especially in females. These data suggested that NAFLD was a much stronger indicator of metabolic diseases than obesity. The traditional view was that obese populations were more prone to MetS than the nonobese, but our study suggested that subjects with NAFLD, even if they were not obese, should be more concerned about the presence of metabolic diseases. Therefore, NAFLD might be better identified as a criterion for the diagnosis of MetS (44), especially for the nonobese subjects who frequently lack of abdominal obesity.

Insulin resistance was presented as a critical node between NAFLD and MetS (45). However, we found 
that the levels of HOMA-IR were comparable between nonobese subjects with NAFLD and obese subjects without NAFLD, which implied that the different risks for metabolic diseases between these two groups were independent of insulin resistance. Future studies are needed to identify the risk factors for metabolic abnormalities in the nonobese subjects with NAFLD. A better understanding of the potential mechanism linking nonobese NAFLD with MetS is urgently required.

There were some limitations in the study. First, in our study, the diagnosis of NAFLD was depended on ultrasonography, which might result in false-negative or false-positive results. However, it was impractical and unnecessary to perform liver biopsy in all suspected NAFLD subjects since it was an invasive procedure (37). Second, there were still sampling biases, although the threestep sampling method was used during selection. Third, we included only residents in Shanghai in this study, which is one of the largest and developed modernized cities in China. Thus, the results might not be generalized to the whole population in China.

In conclusion, we found that the prevalence of NAFLD, obesity and its related diseases in the general population of Shanghai increased considerably during the past decade. Meanwhile, nonobese subjects were more vulnerable to NAFLD as the increase of age and WC than the obese, in particular in females. Although without the phenotype of obesity, the presence of NAFLD in nonobese subjects significantly increased the risk for metabolic diseases than obese subjects without NAFLD.

\section{Acknowledgments}

Funding: This work was supported by grants from the Three-year Action Program of Shanghai Municipality for Strengthening the Construction of the Public Health System (2015-2017) (No. GWIV-27.7).

\section{Footnote}

Reporting Checklist: The authors have completed the STROBE reporting checklist. Available at https://hbsn. amegroups.com/article/view/10.21037/hbsn-20-263/rc

Data Sharing Statement: Available at https://hbsn. amegroups.com/article/view/10.21037/hbsn-20-263/dss

Conflicts of Interest: All authors have completed the ICMJE uniform disclosure form (available at https://hbsn.amegroups. com/article/view/10.21037/hbsn-20-263/coif). The authors report grants from Shanghai Municipal Finance Bureau, during the conduct of the study. The authors have no other conflicts of interest to declare.

Ethical Statement: The authors are accountable for all aspects of the work in ensuring that questions related to the accuracy or integrity of any part of the work are appropriately investigated and resolved. The study was conducted in accordance with the Declaration of Helsinki (as revised in 2013). Written informed consent was obtained from all participants in the study. The study protocol was approved by the Shanghai Changzheng Hospital Institutional Ethics Committee (2016SL039).

Open Access Statement: This is an Open Access article distributed in accordance with the Creative Commons Attribution-NonCommercial-NoDerivs 4.0 International License (CC BY-NC-ND 4.0), which permits the noncommercial replication and distribution of the article with the strict proviso that no changes or edits are made and the original work is properly cited (including links to both the formal publication through the relevant DOI and the license). See: https://creativecommons.org/licenses/by-nc-nd/4.0/.

\section{References}

1. Younossi ZM, Koenig AB, Abdelatif D, et al. Global epidemiology of nonalcoholic fatty liver disease-Metaanalytic assessment of prevalence, incidence, and outcomes. Hepatology 2016;64:73-84.

2. Younossi Z, Anstee QM, Marietti M, et al. Global burden of NAFLD and NASH: trends, predictions, risk factors and prevention. Nat Rev Gastroenterol Hepatol 2018;15:11-20.

3. Li J, Zou B, Yeo YH, et al. Prevalence, incidence, and outcome of non-alcoholic fatty liver disease in Asia, 19992019: a systematic review and meta-analysis. Lancet Gastroenterol Hepatol 2019;4:389-98.

4. De Roza MA, Goh GB. The increasing clinical burden of NAFLD in Asia. Lancet Gastroenterol Hepatol 2019;4:333-4.

5. Fan JG, Kim SU, Wong VW. New trends on obesity and NAFLD in Asia. J Hepatol 2017;67:862-73.

6. Fan JG, Farrell GC. Epidemiology of non-alcoholic fatty liver disease in China. J Hepatol 2009;50:204-10.

7. Marchesini G, Day C, Dufour JF, et al. EASL-EASD- 
EASO Clinical Practice Guidelines for the management of non-alcoholic fatty liver disease. J Hepatol 2016;64:1388-402.

8. Hernandez-Rodas MC, Valenzuela R, Videla LA. Relevant Aspects of Nutritional and Dietary Interventions in Non-Alcoholic Fatty Liver Disease. Int J Mol Sci 2015;16:25168-98.

9. Abenavoli L, Boccuto L, Federico A, et al. Diet and NonAlcoholic Fatty Liver Disease: The Mediterranean Way. Int J Environ Res Public Health 2019;16:3011.

10. Kim D, Kim WR. Nonobese fatty liver disease. Clin Gastroenterol Hepatol 2017;15:474-85.

11. Wei JL, Leung JC, Loong TC, et al. Prevalence and severity of nonalcoholic fatty liver disease in nonobese patients: a population study using protonmagnetic resonance spectroscopy. Am J Gastroenterol 2015;110:1306-14.

12. $\mathrm{Xu} \mathrm{C}, \mathrm{Yu} \mathrm{C}, \mathrm{Ma} \mathrm{H}$, et al. Prevalence and risk factors for the development of nonalcoholic fatty liver disease in a nonobese Chinese population: the Zhejiang Zhenhai Study. Am J Gastroenterol 2013;108:1299-304.

13. Sinn DH, Gwak GY, Park HN, et al. Ultrasonographically detected non-alcoholic fatty liver disease is an independent predictor for identifying patients with insulin resistance in non-obese, non-diabetic middle-aged Asian adults. Am J Gastroenterol 2012;107:561-7.

14. Omagari K, Kadokawa Y, Masuda J, et al. Fatty liver in non-alcoholic non-overweight Japanese adults: incidence and clinical characteristics. J Gastroenterol Hepatol 2002;17:1098-105.

15. Feldman A, Eder SK, Felder TK, et al. Clinical and metabolic characterization of lean caucasian subjects with non-alcoholic fatty liver. Am J Gastroenterol 2017;112:102-10.

16. Sookoian S, Pirola CJ. Systematic review with metaanalysis: the significance of histological disease severity in lean patients with nonalcoholic fatty liver disease. Aliment Pharmacol Ther 2018;47:16-25.

17. Leung JC, Loong TC, Wei JL, et al. Histological severity and clinical outcomes of nonalcoholic fatty liver disease in nonobese patients. Hepatology 2017;65:54-64.

18. Denkmayr L, Feldman A, Stechemesser L, et al. Lean Patients with Non-Alcoholic Fatty Liver Disease Have a Severe Histological Phenotype Similar to Obese Patients. J Clin Med 2018;7:562.

19. Ampuero J, Aller R, Gallego-Durán R, et al. The effects of metabolic status on non-alcoholic fatty liver diseaserelated outcomes, beyond the presence of obesity. Aliment
Pharmacol Ther 2018;48:1260-70.

20. VanWagner LB, Armstrong MJ. Lean NAFLD: A not so benign condition? Hepatol Commun 2018;2:5-8.

21. Adams LA, Anstee QM, Tilg H, et al. Non-alcoholic fatty liver disease and its relationship with cardiovascular disease and other extrahepatic diseases. Gut 2017;66:1138-53.

22. Yki-Järvinen $H$. Non-alcoholic fatty liver disease as a cause and a consequence of metabolic syndrome. Lancet Diabetes Endocrinol 2014;2:901-10.

23. Sookoian S, Pirola CJ. Systematic review with metaanalysis: risk factors for non-alcoholic fatty liver disease suggest a shared altered metabolic and cardiovascular profile between lean and obese patients. Aliment Pharmacol Ther 2017;46:85-95.

24. Mellor TE, Torres DM. Editorial: lean and obese NAFLD-similar siblings. Aliment Pharmacol Ther 2017;46:549-50.

25. Fracanzani AL, Petta S, Lombardi R, et al. Liver and cardiovascular damage in patients with lean nonalcoholic fatty liver disease, and association with visceral obesity. Clin Gastroenterol Hepatol 2017;15:1604-11.e1.

26. Kwon YM, Oh SW, Hwang SS, et al. Association of nonalcoholic fatty liver disease with components of metabolic syndrome according to body mass index in Korean adults. Am J Gastroenterol 2012;107:1852-8.

27. Chinese Diabetes Society. China Guideline for Type 2 Diabetes (2017 Edition, in Chinese). Chin J Diabetes 2018;10:4-66.

28. Alberti KG, Zimmet P, Shaw J. The metabolic syndrome-a new worldwide definition. Lancet 2005;366:1059-62.

29. Fan JG, Zhu J, Li XJ, et al. Prevalence of and risk factors for fatty liver in a general population of Shanghai, China. J Hepatol 2005;43:508-14.

30. Tanaka N, Takahashi S, Matsubara T, et al. Adipocytespecific disruption of fat-specific protein 27 causes hepatosteatosis and insulin resistance in high-fat diet-fed mice. J Biol Chem 2015;290:3092-105.

31. Jin YJ, Kim KM, Hwang S, et al. Exercise and diet modification in non-obese non-alcoholic fatty liver disease: analysis of biopsies of living liver donors. J Gastroenterol Hepatol 2012;27:1341-7.

32. Wong VW, Wong GL, Chan RS, et al. Beneficial effects of lifestyle intervention in non-obese patients with nonalcoholic fatty liver disease. J Hepatol 2018;69:1349-56.

33. Shao C, Ye J, Li F, et al. Different predictors of steatosis and fibrosis severity among lean, overweight and obese patients with nonalcoholic fatty liver disease. Dig Liver Dis 2019;51:1392-9. 
34. Okada R, Yasuda Y, Tsushita K, et al. Upper-normal waist circumference is a risk marker for metabolic syndrome in normal-weight subjects. Nutr Metab Cardiovasc Dis 2016;26:67-76.

35. Sun Y, Liu B, Snetselaar LG, et al. Association of NormalWeight Central Obesity With All-Cause and CauseSpecific Mortality Among Postmenopausal Women. JAMA Netw Open 2019;2:e197337.

36. Després JP, Lemieux I, Bergeron J, et al. Abdominal obesity and the metabolic syndrome: contribution to global cardiometabolic risk. Arterioscler Thromb Vasc Biol 2008;28:1039-49.

37. Chalasani N, Younossi Z, Lavine JE, et al. The diagnosis and management of nonalcoholic fatty liver disease: Practice guidance from the American Association for the Study of Liver Diseases. Hepatology 2018;67:328-57.

38. Lonardo A, Nascimbeni F, Ballestri S, et al. Sex Differences in NAFLD: State of the Art and Identification of Research Gaps. Hepatology 2019;70:1457-69.

39. Tchernof A, Després JP. Pathophysiology of human visceral obesity: an update. Physiol Rev 2013;93:359-404.

Cite this article as: $\mathrm{Hu} P F$, Zeng $\mathrm{X}$, Zou ZY, Tang $W$, Guo YB, Yuan ZL, Shi PM, Tan Y, Song Y, Shi YQ, Xie WF. The presence of NAFLD in nonobese subjects increased the risk of metabolic abnormalities than obese subjects without NAFLD: a population-based cross-sectional study. HepatoBiliary Surg Nutr 2021;10(6):811-824. doi: 10.21037/ hbsn-20-263
40. Yang JD, Abdelmalek MF, Pang H, et al. Gender and menopause impact severity of fibrosis among patients with nonalcoholic steatohepatitis. Hepatology 2014;59:1406-14.

41. McKenzie J, Fisher BM, Jaap AJ, et al. Effects of HRT on liver enzyme levels in women with type 2 diabetes: a randomized placebo-controlled trial. Clin Endocrinol (Oxf) 2006;65:40-4.

42. Lonardo A, Ballestri S, Marchesini G, et al. Nonalcoholic fatty liver disease: a precursor of the metabolic syndrome. Dig Liver Dis 2015;47:181-90.

43. Fukuda T, Hamaguchi M, Kojima T, et al. The impact of non-alcoholic fatty liver disease on incident type 2 diabetes mellitus in non-overweight individuals. Liver Int 2016;36:275-83.

44. Tarantino G, Finelli C. What about non-alcoholic fatty liver disease as a new criterion to define metabolic syndrome? World J Gastroenterol 2013;19:3375-84.

45. Asrih M, Jornayvaz FR. Metabolic syndrome and nonalcoholic fatty liver disease: Is insulin resistance the link? Mol Cell Endocrinol 2015;418:55-65. 
Supplementary

Table S1 Characteristics of the participants with and without NAFLD stratified by gender and BMI

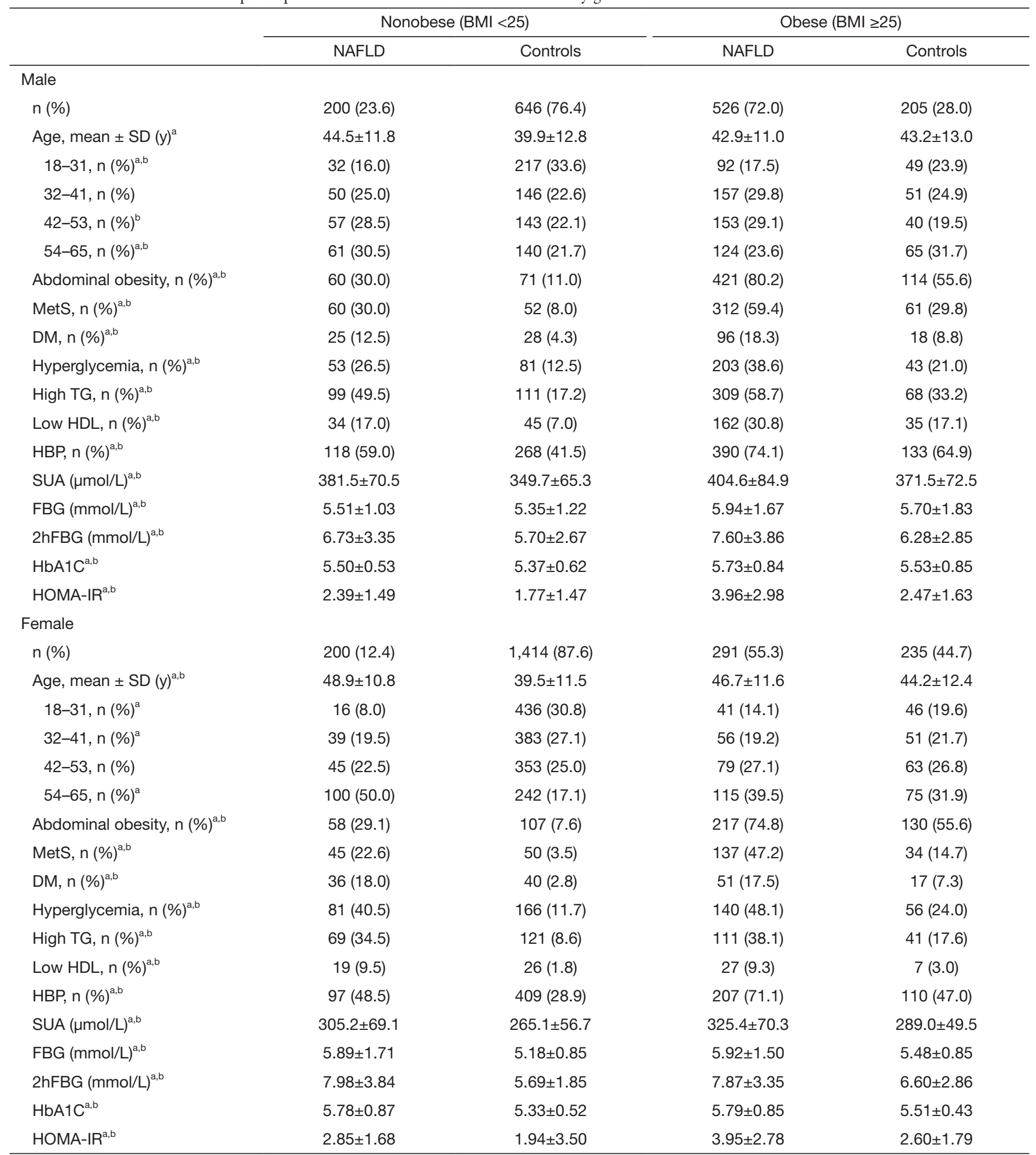

Data are presented as $\mathrm{n}(\%)$ or mean \pm SD. ${ }^{a}$ component with a $\mathrm{P}$ value of $<0.05$ in the normal/overweight group; ${ }^{\mathrm{b}} \mathrm{component}$ with a $P$ value of $<0.05$ in the obese group. NAFLD, nonalcoholic fatty liver disease; SD, standard deviation; BMI, body mass index; MetS, metabolic syndrome; DM, diabetes mellitus; high TG, hypertriglyceridemia; HDL, high-density lipoprotein cholesterol; HBP, high blood pressure; SUA, serum uric acid; FBG, fasting blood glucose; 2hFBG, 2-hour postprandial blood glucose; HOMA-IR, homeostasis metabolic assessment insulin resistance index; $\mathrm{HbA} 1 \mathrm{C}$, hemoglobin A1c. 
Male

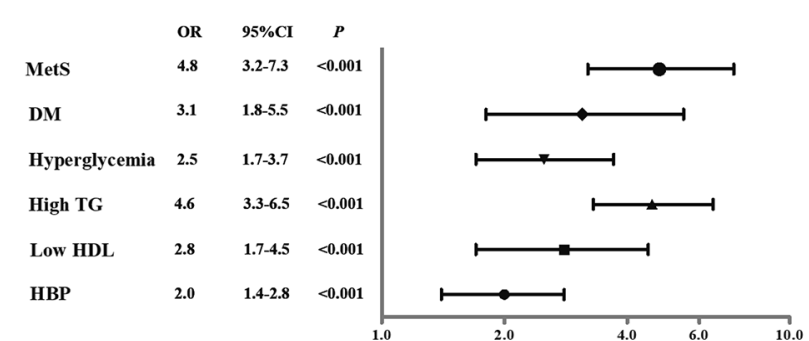

Female

nonobese NAFLD vs nonobese non-NAFLD

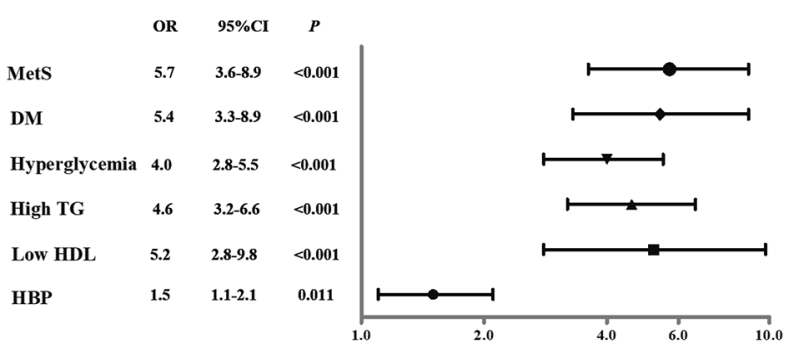

\section{obese NAFLD vs obese non-NAFLD}

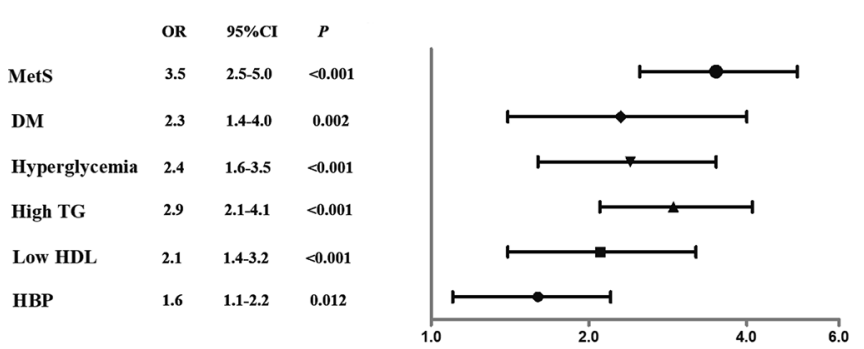

obese NAFLD vs obese non-NAFLD

$\begin{array}{lccc} & \text { OR } & 95 \% \mathrm{CI} & P \\ \text { MetS } & 5.6 & 3.6-8.7 & <0.001 \\ \text { DM } & 2.7 & 1.5-4.8 & 0.001 \\ \text { Hyperglycemia } & 2.9 & 2.0-4.3 & <0.001 \\ \text { High TG } & 2.8 & 1.9-4.3 & <0.001 \\ \text { Low HDL } & 3.3 & 1.4-7.8 & 0.006 \\ \text { HBP } & 2.8 & 1.9-4.1 & <0.001\end{array}$

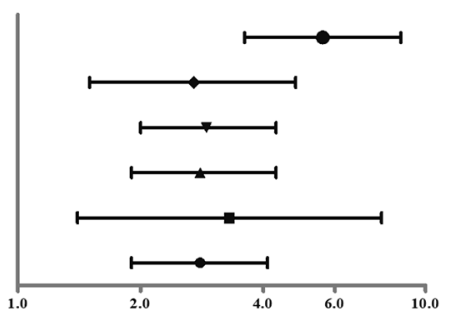

Figure S1 The presence of NAFLD increased the risk of metabolic diseases. According to BMI and the presence of NAFLD or not, we classified the subjects into nonobese subjects without NAFLD (nonobese non-NAFLD), nonobese subjects with NAFLD (nonobese NAFLD), obese subjects without NAFLD (obese non-NAFLD) and obese subjects with NAFLD (obese NAFLD). The presence of NAFLD on the risk for metabolic diseases including metabolic syndrome (MetS), diabetes mellitus (DM), hyperglycemia, hypertriglyceridemia (high TG), low high-density lipoprotein cholesterol (low HDL) and high blood pressure (HBP) were evaluated using a logistic regression model, adjusting for potentially confounding variables, including education, living area, smoking, drinking status and menopause status for females. Data are presented as the odds ratio (OR, 95\% CI). 



Figure S2 The presence of NAFLD in youth increased the risk of metabolic diseases than the older subjects. According to age, we classified the subjects into younger than 45 years (A) or older than 45 years (B). The presence of NAFLD on the risk for metabolic diseases including metabolic syndrome (MetS), diabetes mellitus (DM), hyperglycemia, hypertriglyceridemia (high TG), low highdensity lipoprotein cholesterol (low HDL) and high blood pressure (HBP) were evaluated using a logistic regression model, adjusting for potentially confounding variables, including gender, education, living area, smoking and drinking status. Data are presented as the odds ratio (OR, 95\% CI). 